\title{
New Application, Development and Aerospace Prospect of fNIR
}

\author{
Jinjin Pan, Xuejun Jiao \\ National Key Laboratory of Human Factor Engineering, China Astronaut Research and Training Centre, Beijing, China \\ Email: winston331@126.com
}

Received 2013

\begin{abstract}
Functional near-infrared imaging (fNIR) is a non-invasive, convenient, safe and stable imaging method to test biological state. It can obtain the biological tissue hemodynamic data, thus becoming a powerful tool to measure brain activities, mental workload, metabolism and cognitive activities state. First of all, we introduced the characteristics and current situation of fNIR in this article. Then we focused on the applications of fNIR, discussed some existing problems and future directions, including the prospect in aerospace field. Our purpose is to give a comprehensive description of fNIR and show its potential in aerospace field.
\end{abstract}

Keywords: fNIR; Brain Activity; Hemodynamics; Neuro-imaging Technology

\section{Introduction}

Neural science develops rapidly in recent years. Lots of researches in human cognition and behavior performance have been carried out. Neuroscience research field is very wide, including nervous system structure, nervous function, pathological and so on. Among these aspects, the researches on nervous structure and function are key and fundamental. So neural imaging technology is very important. It developed rapidly these years. And there are several technology methods can be used to observe brain structure and activity. FNIR technology is an excellent neural imaging method of brain observation. It can observe biological state effectively, non-invasively, and easily.

\subsection{Characteristics of fNIR}

FNIR is a newly developed technology that utilizes light from $700 \mathrm{~nm}$ to $900 \mathrm{~nm}$ to monitor the state of biological tissue. This technique can be portable, safe, relatively cheap and noninvasive. [1,2]

It was firstly introduced by Jobsis[24] in his research paper that relatively high degree of brain tissue transparency in the NIR range enables the real-time non-invasive detection of hemoglobin $(\mathrm{Hb})$ oxygenation using transillumination spectroscopy. For about ten years later, this technology had been remarkable developed. Researchers found that the near infrared spectroscopy could be used to monitor brain functional activities, such as monitoring cognitive activities in normal condition and war environment. [3] Early researchers carried out some simple exercise and cognitive tasks, to verify this possibility used for non-invasive monitoring of brain regional activities. Some researchers later began to try to monitor some complex cognitive tasks, such as war management [4] and planes landing simulation [5]. Chance [6], Hoshi [7], and Villringer [8] are firstly proved the possibility of NIR's brain activity monitoring.

\subsection{Principle}

The wavelength range of fNIR is $700 \mathrm{~nm}$ to $900 \mathrm{~nm}$. Most biological tissues are relatively transparent to light in this wavelength, so relatively little scattering of photons occurs when these wavelengths are introduced to tissue. It makes fNIR very suitable for tissue imaging. [1] The main chromophores in the optical window of 600 to $900 \mathrm{~nm}$ are oxy- and deoxy-hemoglobin (denoted HbO and $\mathrm{Hb}$ respectively), water, lipids and cytochrome-coxidase. $\mathrm{HbO}$ and $\mathrm{Hb}$ are mainly of interest because they are related to the regional cerebral blood flow. [9] Using the modified Beer-Lambert law and fNIR measurements conducted at two different wavelengths within the near infrared light range at two adjacent times, the relative changes in concentrations of deoxy-and oxy-Hb can be obtained. [2] These physiological parameters are closely related with human oxidation and metabolism, and can be used for the detection of cognitive activities and mental workload, so fNIR is a ideal tool to detect cerebral cortex function.[4]

There are several neuroimaging methods can realize the monitoring of brain structure and function. They can 
be divided into invasive and non-invasive imaging methods on the basis of whether harmful or not to human body. At present, some high-resolution mode require invasive methods or injection of pharmacological (such as CT, MRI), these are not ideal choice. So many invasive neuroimaging methods become research focus, such as fNIR, EEG, fMRI, MEG, PET etc. [10] Among these techniques, fMRI, EEG and PET are widely used.

Compared with other existing neuroscience imaging methods, fNIR has several advantages. First of all, fNIR can provide some physiological parameter information while other methods cannot, such as oxidation information. In addition, from Figure 1, we can find fNIR has a better time resolution compared with fMRI and PET. FNIR and fMRI can both provide hemodynamic responses information, but the fMRI measurements have much restriction, its equipment is very large. The subjects need supine measurement, and the image acquisition time is long. However, fNIR can acquire data quickly, its time resolution is very high, but it cannot replace fMRI in the aspect of spatial resolution. [11] Compared with EEG, fNIR can offer higher spatial resolution for better observing brain structure. At the same time, fNIR is less susceptible to motion artifacts than EEG. Meanwhile it does not rely on ionizing radiation, therefore it is safer than PET. Some continue-wave fNIR equipment system is very portable, and can be used for telemeasurement. [10,11] In conclusion, fNIR is safe, convenient, unrestricted, green, quick, and balanced. The subjects have no body limitation, and can realize telemeasurement. Especially, its balanced characteristics of time and spatial resolution, anti-jamming and portable advantages make it quite suitable for application in the relative field.

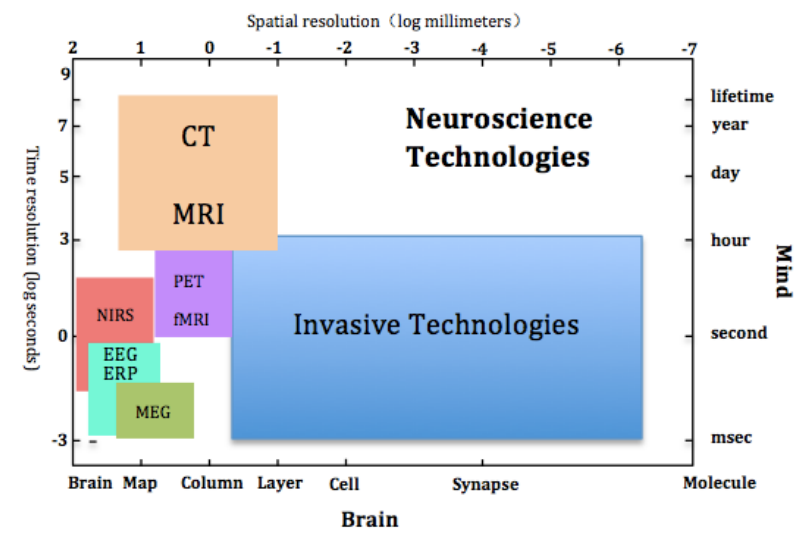

Figure 1. Comparisons of neuroscience technologies [30].

\section{Application of $\mathbf{f N I R}$}

Because of the advantages of fNIR, it develops rapidly in recent years. The researchers take full advantage of fNIR, study and produce many relative applications. Especially in the near year, with the development of neuroscience, some new and advanced applications appeared. FNIR application fields include brain structure and function research, brain-computer-interface, adaptive interface, mental workload assessment, monitoring of newborn, mental fatigue, depth of anesthesia monitoring, medical rehabilitation, cognitive enhancement etc as shown in Table 1.

Table 1. Main fields of fnir applications [28]

\begin{tabular}{ll}
\hline Neurology & - Alzheimer's disease \\
- Dementia & - Depression \\
- Epilepsy \\
- Parkinson's disease \\
- Post-neuro surgery disfunction \\
- Rehabilitation \\
- Stroke recovery \\
- Anxiety disorders \\
- Childhood disorders \\
- Eating disorders \\
- Mood disorders \\
- Personality disorders \\
- Substances related disorders \\
- Schizophrenic disorders \\
- Attention \\
- Body representation \\
- Comprehension \\
- Developmental disorders \\
- Developmental psychology \\
- Emotion \\
- Functional connectivity \\
- Gender differences \\
- Language \\
- Memory \\
- Perception \\
- Reasoning \\
- Social brain \\
- Brain computer interface \\
- Fusion \\
- Neuroergonomics \\
- Pain research \\
- Sleep research \\
- Sports sciences research \\
\hline
\end{tabular}

\subsection{Brain-computer-interface (BCI)}

BCI is an interface system which can obtain signal from the brain, and control the external device or computer through the signal. Application of fNIR in BCI is a relatively new method, it can be used to help evaluate the attention and cognitive load. [12] EEG, fNIR and other methods can be used comprehensively in BCI to evaluate the cognitive state based on neurophysiology and physiology, [13] and to monitor cognitive activities such as attention, working memory, motor nerve activity in clinical and natural conditions. [14]

\subsection{Cognitive Load and Professional Skills Measurement}

Here is an example research of monitoring professional skill development and cognitive load. Kurtulus and several researchers monitored subjects' cognitive load 
and development of professional skill in a simulation UAV environment. The monitor system used was a 16-channel cw-fNIR system. The results showed that the operators' total HB decreased significantly when they transited from beginner to professional status. At the same time, they believed that fNIR had great potential application in research and production, and could also be used to help optimize performance in adverse circumstances.[15] Hasan Ayaz et al also conducted same UAV test, and they got the similar results.

\subsection{Mental Workload Assessment}

Human mental workload plays a very important role in many complex control systems. Villringer.A proved that under natural conditions, fNIR could be used for the evaluation of mental workload in standard experiment (n-back) and complex cognitive tasks (ATC). They also showed that fNIR can evaluate the operators' skill progress in a complex cognitive task. They regarded N-back experiment as a reference to verify the availability of fNIR application in mental workload. They used the 16 channels' data to calculate oxidation information based on MBLL. The results further proved that the degree of expertise indeed affect the prefrontal cortex (PFC) hemodynamic changes of left back.[16]

\subsection{Mental Fatigue Research}

Jiao xuejun and several researchers simulated a mircrogravity environment, and studied the relationship between fNIR sample entropy and power spectrum in normal and fatigue states. The results showed that fNIR signal sample entropy and power spectral entropy decreased obviously during fatigue status. So fNIR has the potential to monitor mental fatigue.[17]

\subsection{Medical Rehabilitation}

FNIR can be used to help monitor cognitive diseases rehabilitation.[18] There were dozens of years since fNIR's first application in monitoring of brain oxidation and metabolism. However, fNIR's history as a mean of monitoring cognitive process and helping monitor nerve repair is relatively short, only 10 years. Similarly to fMRI, the output of fNIR can also be used to produce a brain activation map, thus can be compared by some software. Patricia $\mathrm{M}$ described several application examples in nerve recovery field. These examples showed that fNIR could be applied to monitor brain function recovery after impact effectively, as well as evaluation of brain iniury (TBI) function. He also proposed that a series of united and standard measurement methods were necessary. And we also need to further develop connection device, in order to achieve an integration of a variety of functions. [18] Study of Kurtulus Izzetoglu showed that fNIR could be used to tell the difference between healthy people and patients with TBI.[12] Darren Roblyer conducted a research related to breast cancer, the results showed obvious changes of $\mathrm{Hb}$ after treatment. So we can quickly determine the possible reaction source by using fNIR to monitor the changes or alter the breast cancer treatment plan.[19]

\subsection{Brain Functional Imaging of Newborn}

There was about 10 years since fNIR's first application in newborn brain functional imaging. S. Lloyd-Fox summarized the development of fNIR application in newborn brain functional imaging, and had summed up 38 applications in this field just up to the end of 2009.[11]Fengyu and JiaoXuejun from Tsinghua university and ACC developed a multichannel fNIR system, the system was applicable to neonatal head blood oxygen state bedside monitor. After ensuring system safety, they verified the effectiveness of the system through forearm occlusion and ValSalva's experiments. The experimental results accorded with physical laws, were similar with the relatively results other researchers got, and supplied the blood volume (CBV) information. At the same time, they carried on ValSalva's experiment on adults, which proved the effectiveness of the apparatus for measuring the human head CBV. Their research provided a basis for the further study of neonatal head blood oxygen activity and brain response characteristics, and will promote the study in early diagnosis of neonatal hypoxic ischemic encephalopathy. [20]

\subsection{Pediatric Pain Assessment}

Dr Harel Rosen et al designed a set of pediatric pain assessment by using fNIR. They found obvious growth of oxy-Hb and deoxy-Hb when during pain stimulation. The head blood volume growth under stimulation they showed was consistent with the prior research.[12]

\subsection{Muscle and Brain Oxidation Process Monitor}

Related researchers tested and verified that fNIR can be used for understanding the metabolism process of healthy muscle, the effects of disease on muscle's oxidation and metabolism, and evaluating the efficiency of intervention treatment.[21] Especially in the field of aerospace, muscle oxygen monitoring can be used for guiding the training of athletes, and the astronaut muscle strength change mechanism analysis.

\subsection{Depth of Anesthesia Monitor}


School of medicine in Drexel University conducted an experiment about monitoring depth of anesthesia by fNIR. The results showed that the content of deoxy-Hb had obvious difference between deep and superficial anesthesia, thus proved that fNIR could be used for clinical anesthesia depth monitoring. [12]

\subsection{Social Science and Medicine in the Future}

Relevant researcher focuses on the application of fNIR in neonatal and adult language processing. [28] And there are also some research directions are about the brain responses of social people.[27] Andreas Fallgatter is carrying out a research about psychiatry using fNIR.[28] these research directions of fNIR will contribute to the development of social science and medicine. With the development of fNIR, it can also be used in learning environment. It will help constitute personalized training mechanism, and evaluate the operators' effort in multiple tasks circumstance.[29]

\subsection{Space Environment}

In space environment, fNIR can be used for astronauts to monitor their working load and cognitive performance. Many studies showed that in space environment, the cognition and operation ability of astronauts will change. Study of Stefan Schneider et al demonstrated that fNIR could provide brain structure and functional information in extreme circumstances. And we could use fNIR to explore oxidation information in different regions of the cerebral cortex. So we could realize the measurement of human brain hemodynamic data and function information in space environment.[26]

There are two available methods to measure brain functions in aerospace field, EEG and fNIR. And fNIR has many advantages when compared with EEG. FNIR do not use to apply conductive paste, is not sensitive to motion interference, and is comfortable to objects etc. There are many application fields in apace environment, such as cognition function, mental workload, emotion, adaptive human-machine interaction, training performance assessment in orbit, mental fatigue, operating force varying mechanism analysis and so on.

\section{Problems and Development Trend of fNIR}

FNIR has lots of advantages, such as safe, convenient, little restriction, fast imaging, green, it has balanced time and spatial revolution, and it can be used freely not only in daily life, but also in extreme circumstance like space environment. However, it still exist some problems that need further to develop, such as MA (motion aircraft), physiological noise, resolution, unified analytical method and so on.[25]

\subsection{Motion aircraft (MA)}

FNIR can monitor the changes of $\mathrm{HbO}$ and $\mathrm{Hb}$ in the brain. However, any physiological phenomenon or activity occurred from head or other parts of the body, can lead to changes in the fNIR indexes, and bring the fNIR signals some interference. In particular, the motion of human body can lead to this change, which is called MA. Eliminating the interference of human motion on fNIR signal, is very necessary for the reliable use of cognitive activity evaluation.[22]

Some researchers are doing related study about fNIR MA, Meltem Izzetoglu proposed a Kalman filter to solve the movement interference for fNIR signals. In fNIR field, there are only adaptive filer and Wiener filter methods before, which were widely used in biology, communication, and voice processing etc. However, adaptive filter needs additional sensor and hardware, and changes the transmission function according to the characteristics of input signals. And Wiener filter need fixed data, it cannot be effectively used in real-time environment. This newly proposed method could reduce the motion interference in fNIR signal effectively. FNIR, which combines the advantages of adaptive filter with the Wiener filter, has higher SNR, needs no extra sensor, and is applicable for real-time use. [23]

In addition, Behnam Molavi proposed a fNIR MA elimination method based on wavelet. This method relies on the difference of cycle and amplitude between motion artifacts and fNIR signals, and is designed specially for long motion artifacts. Through fNIR experiments on 3 infants, they confirmed that this method could effectively eliminate the motion artifacts, and obtain a better artifact reduction. This method is very suitable for eliminating fringy motion artifacts, and artifacts with short cycle and high amplitude. And the wavelets are changed adaptively based on the changes of noise. [22]

These methods are effective, but they also have some limitations. Therefore, one development direction of fNIR signal should be to minimize interference as weak as possible. Little MA is an advantage for fNIR compared with other imaging methods (such as EEG, fMRI etc), so it is important to constantly develop and strengthen this advantage.

\subsection{Resolution Problem}

From fig [1] we can see that, fNIR has higher spatial resolution than EEG, and higher time resolution than fMRI. It is a balanced and comprehensive method. However, fNIR may be less excellent than EEG or fMRI in some extreme required conditions, such as in very high spatial or time resolution conditions. Therefore, we should combine and use their advantages. A very good method is to combine the fNIR with EEG or fMRI, make full use of their advantages, so can we deal with the 
resolution problem and get accurate and quick brain information presentation.

\subsection{Sensor}

Compared to the existing fixed hood type fNIR sensor, future design of sensor should include modular sensor and hairbrush sensor. The modular sensor can adapt to adult and children, and all kinds of forehead size. And hairbrush sensor can install light source and perceptron in the hairbrush, it can make the light source and detector contacted to skin directly by separated the hair. This design will allow fNIR to measure information of forehead and all other cortex area in brain, which has never been realized before.[2] And we should also focus on the issue about how to detect the comfort and accuracy of sensor.

\subsection{Develop toward More Convenient Direction}

FNIR develops from wired style to wireless style. There was only cable fNIR equipment before Maurico Rodriguez published an article about wireless fNIR device in 2011. He studied and designed a wireless fNIR system. Science develops quickly, and now we can find many excellent wireless fNIR products. [1]

Although fNIR is very smart and convenient when compared with other imaging modalities, it still needs to develop toward a more convenient, compact, and light direction. Thus it can be more suitable for using in learning and training.

\subsection{Establish a Standardized Method of Data Analysis}

Although we have made remarkable progress in the field of light propagation in brain tissue, and the field of image reconstruction from optical data, there is no standard data analysis method to establish DOT image by multi-channel system, and no standard data analysis method to determine statistical characteristics and cortical oxidative changes.[28] So establishing unified data analysis methods is also a very important development direction for fNIR technology application.

\subsection{Combination with Other Techniques}

By combining the eye-movement apparatus and fNIR technology, we can capture the attention points and get relative brain activities simultaneously. This function can be easily realized by combining convenient fNIR and accurate eye-movement apparatus.[27]

In addition, we can achieve more accurate and quick presentation of brain structure and function by combining
fNIR with EEG, fMRI etc.

\section{Application Prospect of Aerospace}

Neuroscience is developing very rapidly, and fNIR will no doubt play a key role in future as a human brain activity measurement tool in neuroscience, especially in aerospace field.

Aerospace comprises the atmosphere of earth and surrounding space. Typically, aerospace industries combine aeronautics and astronautics to design and maintain vehicles moving through air and space. The environment in aerospace is very extreme, such as weightlessness, radiation, vacuum, large temperature difference, vibrancy, and long distance. Aerospace activities are hard, large and expensive. So each kilogram in weight must be seriously considered in aerospace field.

The equipment used in space circumstance must be portable, safe, simple and no much restriction, and fNIR has these characteristics. Thus fNIR is competitive in the field of aerospace. FNIR may be used to monitor the astronauts' performance and skills during training and learning process. FNIR may also be used to acquire astronauts' cognitive state when they were in space work. To sum up, there are many application fields in apace environment, such as cognition function, mental workload, emotion, adaptive human-machine interaction, training performance assessment in orbit, mental fatigue, operating force varying mechanism analysis and so on.

With the development of science and technology, people pay more attention to human being themselves. We can see more and more human-centric training, learning, and human-centered machine and equipment emerge, and these new things also obey the ergonomics law of safety, efficiency, and comfort.

The technology of fNIR not only obtains human physicology and brain activity information, but also has the characteristics of safe, green, convenient, quick, and ergonomics trait. These proved the considerable development space of fNIR. We can see its fine vitality and development prospect in aerospace and many other fields.

\section{REFERENCES}

[1] A. Illringer and B. Chance, "Non-invasive Optical Spectroscopy and Imaging of Human Brain Function," Trends in Neuroscience, Vol. 1997, No. 20, pp. 435-442 doi:10.1016/S0166-2236(97)01132-6

[2] M. Izzetoglu, K. Izzetoglu, S. Bunce, B. Onaral and K. Pourrezaei, "Functional Near Infrared Neuroimaging," Neural Systems and Rehabilitation Engineering, IEEE Transactions on, Vol. 13, No. 2, 2005, pp. 153-159. 
[3] M. Izzetoglu, A. Devaraj, S. Bunce and B. Onaral, "Motion Artifact Cancellation in NIRs Using Wiener Filtering,” IEEE Trans on BME, Vol. 52, No. 5, 2005, pp. 934-938.

[4] K. Izzetoglu, S. Bunce, M. Izzetoglu, B. Onaral and K. Pourrezaei, "FNIR Spectroscopy As a Measure Of Cognitive Task Load," Proceedings of the 25th Annual International Conference of the IEEE EMBS, 2003, pp. 3431-3434.

[5] Y. Takeuchi, "Change In Blood Volume In The Brain During A Simulated Aircraft Landing Task,” Journal of Occupational Health, Vol. 42, 2000, pp. 60-65.

[6] B. Chance, et al., "Comparison of Time-Resolved and Unresolved Measurement of Deoxy Hemoglobin in Brain," In Proceedings of National Academy of Science, Vol. 1, 1988, pp. 4971-4975.

[7] Y. Hoshiand and M. Tamura, "Detection of Dynamic Changes in Cerebral Oxygenation Coupled to Neuronal Function During Mental Work In Man,” Neuroscience Letters, Vol. 150, 1993, pp. 5-8.

[8] A. Villringer, et al. "Near Infrared Spectroscopy (Nirs): A New Tool to Study Hemodynamic Changes During Activation of Brain Function in Human Adults," Euroscience Letters, Vol. 154, 1993, pp. 101-104.

[9] S. I-Young and Yaz. c. Birsen, "Near Infrared Imaging and Spectroscopy for Brain Activity Monitoring," NATO Security through Science Series, Vol. 2, 2006, pp. 341-372.

[10] "Opportunities in Neuroscience for Future Army Applications," Committee on Opportunities in Neuroscience for Future Army Applications, Vol. 11, 2009, pp. 11-11.

[11] S. Lloyd-Fox, A. Blasi and C. E. Elwell, "Illuminating the Developing Brain: The Past, Present and Future of Functional Near Infrared Spectroscopy,” Neuroscience \& Biobehavioral Reviews, Vol. 34, No. 3, 2010, pp. 269-284.doi:10.1016/j.neubiorev.2009.07.008

[12] Kurtulus, Izzetoglu, et al., "The Evolution of Field Deployable Fnir Spectroscopy from Bench to Clinical Settings," Journal of Innovative Optical Health Sciences, Vol. 4, No. 3, 2011, pp. 239-239. doi:10.1142/S1793545811001587

[13] Dorneich, et al, "Supporting Real-time Cognitive State Classification on a Mobile Individual," Journal of Cognitive Engineering and Decision Making, Vol. 1, No. 3, 2007, pp. 240-270.doi:10.1518/155534307X255618

[14] Mauricio, Rodriguez. Development of a Versatile Wireless fNIR System[J/OL]. http://hdl.handle.net/1860/3733.

[15] Kurtulus, Izzetoglu, Hasan, Ayaz, et al, “Applications of Functional Near Infrared Imaging: Case Study on UAV Ground Controller,” Lecture Notes In Computer Science, Vol. 1, 2011, pp. 608-617.

[16] K. Izzetoglu, S. Bunce, B. Onaral, K. Pourrezaei and B. Chance, "Functional Optical Brain Imaging Using Near-Infrared During Cognitive Tasks,” International Journal of Human-Computer Interaction, Vol. 17, No. 2,
2004, pp. 211-227.doi:10.1207/s15327590ijhc1702_6

[17] X. J. Jiao, J. Bai, S. G. Chen and Q. J. Li, "Browse Conference Publications, Virtual Environments Human-Co," Virtual Environments Human-Computer Interfaces and Measurement Systems (VECIMS), 2012 IEEE International Conference on, Vol. 1, 2012, pp. 74-77.

[18] M. Patricia, Arenth, Joseph, H. Ricker, Maria and T. Schultheis, "Applications of Functional Near-Infrared Spectroscopy (fNIRS) to Neurorehabilitation of Cognitive Disabilities,” The Clinical Neuropsychologist, Vol. 21, No. 1, 2007, pp. 38-57

[19] D. Roblyer, et al., "Optical Imaging of Breast Cancer Oxyhemoglobin Flare Correlates with Neoadjuvant Chemotherapy Response One Day After Starting Treatment," Proceedings of the National Academy of Science of the United States of America, Vol. 108, No. 15, 2011, pp. 14626-14631.

[20] Y. Feng, J. S. Chen, X. J. Jiao and J. Bai, "Design of a Multi-channel Near-Infrared Spectroscopy System Used for Bedside Real-time Monitoring of Newborn Infants' Brain," Space Medicine \& Medical Engineering, Vol. 25, No. 1, 2012, pp. 25-27.

[21] M. Ferrari, T. Binzoni and Quaresima, "Oxidative Metabolism in Muscle,” Philosophical Transactions of the Royal Society B Biological Sciences, Vol. 352, 1997, pp. 677-683.

[22] B. Molavi and G. A. Dumont, "Wavelet-Based Motion Artifact Removal for Functional Near-Infrared Spectroscopy,” Physiological Measurement, Vol. 33, No. 2, 2012, pp. 259-261.

[23] M. Izzetoglu, P. Chitrapu, S. Bunce and B. Onaral, "Motion Artifact Cancellation in NIR Spectroscopy Using Discrete Kalman Filtering[J/OL], BioMedical Engineering On Line.

[24] F. F. Jobsis, "Noninvasive, Infrared Monitoring of Cerebral and Myocardial Oxygen Sufficiency and Circulatory Parameters,” Science, Vol. 198, No. 4323, 1977, pp. 1264-1267.

[25] R. Parasuraman, "Neuroergonomics: Brain, Cognition, and Performance at Work," Current Directions in Psychological Science, Vol. 20, No. 3, 2011, pp. 181-186.

[26] S. Schneider, "Imaging of Neuro-Cognitive Performance in Extreme Environments-A (p) Review,” Planetary and Space Science, Vol. 74, 2012, pp. 135-141.

[27] M. P. Cakir, “A Group Cognitive Perspective on the Multimodal Analysis of Learning," Third International Conference on Emerging Intelligent Data and Web Technologies.

[28] M. Ferrari, “A Brief Review on the History of Human Functional Near-Infrared Spectroscopy (fNIRS) Development and Fields of Application,” NeuroImage, Vol. 63, 2012, pp. 921-935.

[29] H. Ayaz, "Monitoring Expertise Development during Simulated UAV Piloting Tasks using Optical Brain Imaging [J/OL]," Aerospace Conference, 2012 IEEE. 